\title{
Tumour-infiltrating lymphocyte scores effectively stratify outcomes over and above p16 post chemo-radiotherapy in anal cancer
}

\begin{abstract}
Duncan C Gilbert ${ }^{\star}, 1$, Eva Serup-Hansen ${ }^{2}$, Dorte Linnemann ${ }^{3}$, Estrid Høgdall ${ }^{3}$, Charles Bailey ${ }^{4}$, Jeff Summers ${ }^{4}$, Hanne Havsteen ${ }^{2}$ and Gareth J Thomas ${ }^{5,6}$

${ }^{1}$ Sussex Cancer Centre, Royal Sussex County Hospital Eastern Road, Brighton, Sussex BN2 5BE, UK; ${ }^{2}$ Department of Oncology, Herlev Hospital, University of Copenhagen, Herlev Ringvej 75, DK-2730, Denmark; ${ }^{3}$ Department of Pathology, Herlev Hospital, University of Copenhagen, Herlev Ringvej 75, DK-2730, Denmark; ${ }^{4}$ Kent Oncology Centre, Maidstone Hospital, Hermitage Lane, Maidstone, Kent ME16 900, UK; ${ }^{5}$ Department of Cellular Pathology, University Hospital Southampton NHS Foundation Trust, Tremona Road, Southampton SO16 6YD, UK and ${ }^{6}$ Cancer Sciences Unit, University of Southampton, Tremona Road, Southampton SO16 6YD, UK
\end{abstract}

Background: The majority (90\%) of anal cancers are human papillomavirus (HPV)-driven, identified using immunochemistry for p16. Compared with HPV - patients, those with HPV + disease generally show improved survival, although relapse rates around $25 \%$ indicate a need for further stratification of this group.

Methods: Using two cohorts of anal cancer, previously characterised for p16, we assessed the prognostic value of tumourinfiltrating lymphocytes (TILs).

Results: Tumour-infiltrating lymphocyte scores were used to stratify p16+ cases, where tumours with absent/low levels of TIL had a relapse-free rate of $63 \%$, as opposed to $92 \%$ with high levels of TIL (log rank $P=0.006$ ).

Conclusions: Assessment of TIL adds to p16 status in the prognosis of anal cancer following chemo-radiotherapy and provides evidence of the clinical importance of the immune response.

Squamous cell carcinomas of the anus and anal canal are relatively rare tumours, though increasing in incidence for reasons unknown. In keeping with other squamous cell cancers of the ano-genital region and an increasing proportion of oropharyngeal cancer, they are associated with high-risk human papillomavirus (HPV) subtypes (Baracevic-Jones et al, 2015). Immunohistochemistry for $\mathrm{p} 16^{\mathrm{INK} 4 \mathrm{~A}}$ (p16) has been used as a surrogate for HPV involvement and to identify a group of patients (p16 negative) with very poor outcomes following chemo-radiotherapy (CRT) (Gilbert et al, 2013; Koerber et al, 2014; Rodel et al, 2014; Serup-Hansen et al, 2014). Relapse-free rates (RFRs) in p16 + cases were $64-84 \%$ across the four studies as opposed to $35-52 \%$ in p $16-$ cases. High p16 expression correlated with female sex and, in some studies, marginally lower stage at presentation. p16 retained independent prognostic ability in all reported studies, but requires prospective validation in a clinical trial prior to entering routine use (or being used to stratify treatments in future trials). However, rates of p16 positivity ranged from $65 \%$ (Rodel et al, 2014) to $93 \%$ (Serup-Hansen et al, 2014); hence the majority of relapses still occur in p16 + patients.

One biological explanation for the improved outcomes following CRT in p16 + cases rests on intact apoptotic mechanisms, with low rates of p53 mutations described in p16 + head and neck (Westra et al, 2008) and anal cancer (Meulendijks et al, 2015). Human papillomavirus infection uncouples cell cycle checkpoints via suppression of $\mathrm{p} 53 / \mathrm{pRB}$ while retaining wild-type gene

*Correspondence: Dr D Gilbert; E-mail: dcgilbert@doctors.org.uk or duncan.gilbert@bsuh.nhs.uk

Received 27 August 2015; revised 7 October 2015; accepted 30 November 2015; published online 5 January 2016

(c) 2016 Cancer Research UK. All rights reserved 0007-0920/16 
expression, overcome in the case of the dramatic DNA damage that results from combination CRT (Kimple et al, 2013; Reickmann et al, 2013). However, in head and neck cancers, improved survival is also seen in patients with p16 + tumours treated with surgical excision alone, suggesting that further inherent factors are involved (Licitra et al, 2006). Relapse rates of around $25 \%$ in p $16+$ anal cancers suggest that these patients can be further stratified to identify those at 'high risk', with additional mechanisms and biomarkers as yet undiscovered.

An alternative hypothesis is that viral antigens elicit a host immune response directed against the tumour cells. Human papillomavirus 16-E6 and -E7-specific T cells have been identified in HPV-driven oropharyngeal cancer patients as part of an adaptive immune response, and tumour-infiltrating lymphocytes (TILs) are associated with improved outcomes in a number of different tumour types (Mei et al, 2014; Matsumoto et al, 2015). Specifically in the case of HPV-associated oropharyngeal cancer, the degree of TILs can be used to stratify the HPV + patients into 'high-risk' and 'low-risk' groups, where HPV + tumours with only low TIL scores have similar outcomes to the HPV - tumours (Ward et al, 2014). Patients with HPV + /TIL high had a 3-year overall survival of $96 \%$ vs 59\% in the HPV + /TIL low group.

The aim of this study was to investigate whether TILs play a similar prognostic role in anal cancer, using two well-characterised cohorts of tumours (i.e. known p16 status) treated with radical radiotherapy $+1-$ concurrent chemotherapy with curative intent and documented outcomes (Gilbert et al, 2013, Serup-Hansen et al, 2014).

\section{MATERIALS AND METHODS}

Collection of cohorts. This work has ethical approval (11/LO/0323). Clinical details were retrieved for patients treated with radical radiotherapy $+/-$ concurrent chemotherapy with curative intent for non-metastatic squamous cell carcinomas of the anus and anal canal between 2004 and 2009 inclusive at the Sussex Cancer Centre, Brighton, and the Kent Oncology Centre, Maidstone, UK, as previously described (Gilbert et al, 2013). The Danish cohort comprised patients presenting to the Department of Oncology at Herlev Hospital (Herlev, Denmark) with newly diagnosed AJCC stage I-III anal cancer, between January 2000 and January 2010 (Serup-Hansen et al, 2014).

TIL scoring. Haematoxylin and eosin (H\&E) stained slides were obtained from a representative formalin-fixed paraffin-embedded block from each tumour sample. Lymphocyte infiltrations were scored by a pathologist blinded to patient demographics or outcomes (GJT), using a low-power magnification $(\times 2.5$ objective). As described previously (Ward et al, 2014), tumour TILs were scored as high ( 3 - diffuse, present in $>80 \%$ tumour/stroma), moderate ( 2 - patchy, present in $20-80 \%$ tumour/stroma) or low ( 1 - weak/absent, present in $<20 \%$ tumour/stroma).

Statistical analysis. Associations between TIL score and other clinic-pathological characteristics were evaluated using Pearson's correlation coefficient. The primary end point was relapse-free survival, defined as time from histological diagnosis of anal cancer to date of diagnosis of confirmed relapse (local or distant as recorded by the clinical team) or date of last follow-up, analysed using Kaplan-Meier plots and log-rank tests using SPSS 22.0 (IBM, Armonk, NY, USA), tabulating RFRs.

\section{RESULTS}

UK cohort. In all 153 patients were available for analysis (Table 1), with a median follow-up of 27.9 months. Of these,
Table 1. UK and Danish anal cancer cohorts, demographics including p16 immunohistochemistry and quantification of TILs

\begin{tabular}{|c|c|c|}
\hline & UK & Danish \\
\hline$N$ & 153 & 131 \\
\hline Male (\%) & $56(36.6)$ & $33(25.2)$ \\
\hline Female (\%) & $97(63.4)$ & $98(74.8)$ \\
\hline Relapse (RFR\%) & $106(69.3)$ & $33(74.8)$ \\
\hline \multicolumn{3}{|l|}{ p16 } \\
\hline $\begin{array}{l}p 16+(\%) \\
p 16-(\%)\end{array}$ & $\begin{array}{r}137(89.5) \\
16(10.5)\end{array}$ & $\begin{array}{c}121(92.4) \\
10(7.6)\end{array}$ \\
\hline \multicolumn{3}{|l|}{ p16 and relapse } \\
\hline $\begin{array}{l}\text { p16 + relapse (\%RFR) } \\
\text { p16 - relapse (\%RFR) }\end{array}$ & $\begin{array}{r}37 / 137(73.0) \\
10 / 16(37.5) \\
\end{array}$ & $\begin{array}{c}26 / 121(78.5) \\
7 / 10(30)\end{array}$ \\
\hline \multicolumn{3}{|l|}{ TIL } \\
\hline $\begin{array}{l}\text { TIL1 (\%) } \\
\text { TIL2 (\%) } \\
\text { TIL3 (\%) } \\
\text { TIL not available (\%) }\end{array}$ & $\begin{array}{l}29(18.9) \\
85(55.5) \\
27(17.6) \\
12(7.8)\end{array}$ & $\begin{array}{c}23(17.6) \\
73(55.7) \\
26(19.8) \\
9(6.9)\end{array}$ \\
\hline \multicolumn{3}{|l|}{ TIL and relapse } \\
\hline $\begin{array}{l}\text { TIL1 relapse (\%RFR) } \\
\text { TIL2 relapse (\%RFR) } \\
\text { TIL3 relapse (\%RFR) }\end{array}$ & $\begin{array}{r}16 / 29(44.8) \\
23 / 85(72.9) \\
4 / 27(85.2)\end{array}$ & $\begin{array}{r}5 / 23(78.3) \\
25 / 73(65.8) \\
2 / 26(92.3)\end{array}$ \\
\hline \multicolumn{3}{|l|}{ Whole cohort, p16- } \\
\hline $\begin{array}{l}\text { TIL1 relapse (\%RFR) } \\
\text { TIL2 relapse (\%RFR) } \\
\text { TIL3 relapse (\%RFR) }\end{array}$ & \multicolumn{2}{|c|}{$\begin{array}{c}4 / 6(33.3) \\
11 / 16(31.3) \\
2 / 3(33.3)\end{array}$} \\
\hline \multicolumn{3}{|l|}{ Whole cohort, p16+ } \\
\hline $\begin{array}{l}\text { TIL1 relapse (\%RFR) } \\
\text { TIL2 relapse (\%RFR) } \\
\text { TIL3 relapse (\%RFR) }\end{array}$ & $\begin{array}{r}17 \\
37 / \\
4 /\end{array}$ & \\
\hline
\end{tabular}

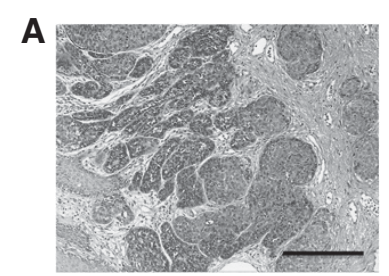

TIL low

B

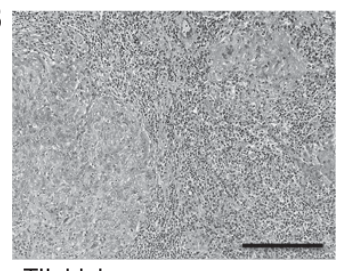

TIL high

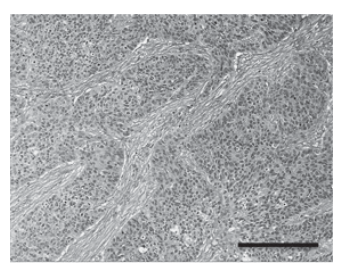

TIL low

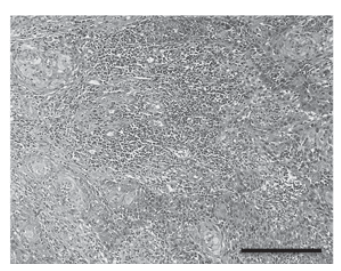

TIL high
Figure 1. Representative anal cancer specimens stained with haematoxylin and eosin demonstrating examples with (A), low/absent and (B), high levels of tumour-infiltrating lymphocytes corresponding to TIL1 and TIL3 respectively. Scale bar denotes $10 \mu \mathrm{m}$.

97 were female and 56 male. Tumour-infiltrating lymphocyte scores were available for 141 cases (representative images; Figure 1); 12 cases were excluded where insufficient tumour material was available. Of these, 126 were p16+ (Gilbert et al, 2013), with an RFR of $73.8 \%$ as opposed to $33.3 \%$ in the 15 p16tumours. There was no correlation between TIL score and patients' sex or p16 immunohistochemistry. 
Tumour-infiltrating lymphocyte score was significantly associated with relapse-free survival (Figure 2A, log-rank test $P=0.004$ ), where tumours with a TIL score 3 had an RFR of $85.2 \%$ as opposed to $72.9 \%$ for TIL2 and $44.8 \%$ for those with weak or absent TILs.

Danish cohort. One hundred thirty-one patients were included in the analysis, with a median follow-up of 51.2 months. Of these, 98 were female and 33 male (Table 1). As previously described (Serup-Hansen et al, 2014), 121 (92.3\%) were positive for p16 immunohistochemistry, with an RFR of $78.5 \%$, as opposed to $30 \%$ in the 10 cases that were p16-. Tumour-infiltrating lymphocyte scores were available for 122 cases; 9 cases were excluded where insufficient tumour material was available. Again there was no correlation between TIL score and sex or p16 immunohistochemistry.

Tumour-infiltrating lymphocyte score was again associated with relapse-free survival (Figure $2 \mathrm{~B}$ and log-rank test $P=0.033$ ), although here the biggest distinction was between TIL3 and TILs 1-2 (weak/absent to moderate). Patients with high TILs had a RFR of $92.3 \%$, as opposed to $65.8 \%$ with a moderate infiltrate of TILs and $78.3 \%$ when TILs were absent or weakly infiltrating.

Combined analysis of cohorts with respect to p16 status. In order to better understand the relative contribution of TIL scoring in the context of p16 status in line with the HNSCC model previously proposed (Ward et al, 2014), the two cohorts were combined and survival analyses performed on the p16+ and p16 - populations (Table 1, Figure 2C and D). Tumourinfiltrating lymphocyte scores did not add prognostic value to the 25 cases that were negative for p16 (log-rank test $P=0.416$ ), but effectively stratified the outcomes in the 263 patients with p16 + tumours (log-rank test $P=0.006$ ), with RFRs ranging from $63 \%$ for the TIL1 cases to $92 \%$ in the TIL3 tumours.
In a multivariate analysis including sex, p16 and TIL score, TIL retained independent prognostic value $(P=0.001)$.

\section{DISCUSSION}

A number of studies have shown that, in keeping with the situation described in head and neck cancer, anal cancers associated with HPV have better outcomes following CRT than those that are HPV or p16-. Previous explanations for this have centred on differential levels of p53 mutation, either directly measured (Meulendijks et al, 2015) or implied through immunohistochemistry for p53 (Lampejo et al, 2010). However, an additional factor might be variations in the adaptive immune response elicited by virally driven tumours. As one component of this, TILs provide a morphological correlate of the host immune response and, as we have demonstrated, may give additional prognostic value over and above assessment of the HPV status of tumours via HPV genotyping or immunohistochemistry for p16. Prior data from anal cancer are limited and inconsistent (Grabenbauer et al, 2006; Rubio et al, 2008). Whether these differences are derived from tumour- or host-related factors will be the subject of further research. Given the high rates of HPV/p16 positivity of 90\% in contemporary anal cancer (Baricevic et al, 2015), the ability of TIL to stratify outcomes in the p16+ population is arguably even more relevant than in head and neck cancer.

Larger data sets are required to demonstrate the reproducibility of TIL score and build a multivariate model incorporating TIL scores with established prognostic factors (p16/HPV, stage, sex, detailed smoking history) and correlate any prognostic effect on overall survival (we used relapse-free survival as salvage surgery is possible for a number of cases). Furthermore, these effects require
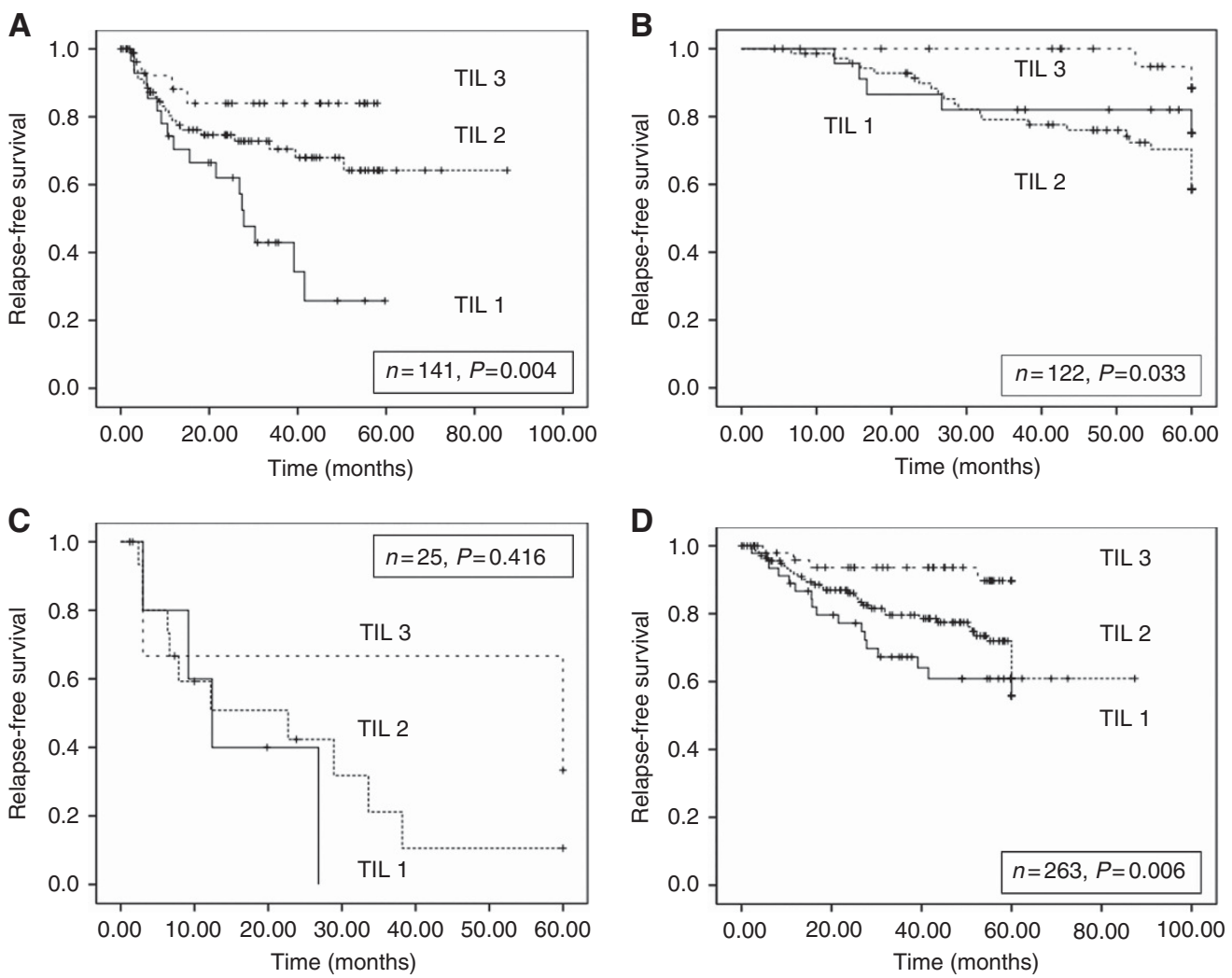

Figure 2. Kaplan-Meier relapse-free survival curves and log rank test $P$ values; (A) For UK cohort stratified by TIL 1-3; (B) for Danish cohort stratified by TIL 1-3; (C) combined cohorts, p16- cases stratified by TIL 1-3; and (D) combined cohorts, p16 + cases stratified by TIL 1-3. 
validation in prospective studies. This work is underway and should inform future trials exploring increased radiotherapy doses or novel agents in poor-risk cases, or 'de-escalation' strategies in good prognosis tumours where avoidance of late toxicity is desirable. The marked biological and clinical relevance of the TIL response demonstrated here supports trials of immunotherapy in anal cancer, whether targeting immune checkpoints via antiCTLA4/PD1/PDL1 agents (including in the concurrent or (neo) adjuvant setting; Illidge, 2015), or using adoptive T-cell therapy as demonstrated in head and neck and cervical cancer (Hald et al, 1994; Stevanović et al, 2015).

\section{ACKNOWLEDGEMENTS}

This work was funded by the Cancer Fund of Brighton and Sussex University Hospitals NHS Trust.

\section{CONFLICT OF INTEREST}

The authors declare no conflict of interest.

\section{REFERENCES}

Baricevic I, He X, Chakrabarty B, Oliver AW, Bailey C, Summers J, Hampson L, Hampson I, Gilbert DC, Renehan AG (2015) High-sensitivity human papilloma virus genotyping reveals near universal positivity in anal squamous cell carcinoma: different implications for vaccine prevention and prognosis. Eur J Cancer 51: 776-785.

Gilbert DC, Williams A, Allan K, Stokoe J, Jackson T, Linsdall S, Bailey CM, Summers J (2013) p16(INK4A), p53, EGFR expression and KRAS mutation status in squamous cell cancers of the anus: Correlation with outcomes following chemo-radiotherapy. Radiother Oncol 109: 146-151.

Grabenbauer GG, Lahmer G, Distel L, Niedobitek G (2006) Tumor-infiltrating cytotoxic $\mathrm{T}$ cells but not regulatory $\mathrm{T}$ cells predict outcome in anal squamous cell carcinoma. Clin Cancer Res 12: 3355-3360.

Hald J, Rasmussen N, Claesson MH (1994) In vivo infiltration of mononuclear cells in squamous cell carcinoma of the head and neck correlated with the ability to expand tumour-infiltrating $\mathrm{T}$ cells in vitro and with the expression of MHC class I antigens on tumour cells. Cancer Immunology, Immunotherapy 39(6): 383-390.

Illidge T (2015) Turning radiotherapy into an effective systemic anti-cancer treatment in combination with immunotherapy. Clin Oncol. 27: 696-699.

Kimple RJ, Smith MA, Blitzer GC, Torres AD, Martin JA, Yang RZ, Peet CR, Lorenz LD, Nickel KP, Klingelhutz AJ, Lambert PF, Hrari PM (2013) Enhanced radiation sensitivity in HPV-positive head and neck cancer. Cancer Research 73(15): 4791-1800.
Koerber SA, Schoneweg C, Slynko A, Krug D, Haefner MF, Herfarth K, Debus J, Sterzing F, von Knebel Doeberitz M, Prigge ES, Reuschenbach M (2014) Influence of human papillomavirus and p16(INK4a) on treatment outcome of patients with anal cancer. Radiother Oncol 113: 331-336.

Lampejo T, Kavanagh D, Clark J, Goldin R, Osborn M, Ziprin P, Cleator S (2010) Prognostic biomarkers in squamous cell carcinoma of the anus: a systematic review. Br J Cancer 103: 1858-1869.

Licitra L, Perrone F, Bossi P, Suardi S, Mariani L, Artusi R, Oggionni M, Rossini C, Cantu G, Squadrelli M, Quattrone P, Locati LD, Bergamini C, Olmi P, Pierotti MA, Pilotti S (2006) High-risk human papillomavirus affects prognosis in patients with surgically treated oropharyngeal squamous cell carcinoma. J Clin Oncol 24(36): 5630-5636.

Matsumoto H, Koo SL, Dent R, Tan PH, Iqbal J (2015) Role of inflammatory infiltrates in triple negative breast cancer. J Clin Pathol 68(7): 506-510.

Mei Z, Liu Y, Liu C, Cui A, Liang Z, Wang G, Peng H, Cui L, Li C (2014) Tumour-infiltrating inflammation and prognosis in colorectal cancer: systematic review and meta-analysis. Br J Cancer. 110(6): 1595-1605.

Meulendijks D, Tomasoa NB, Dewit L, Smits PH, Bakker R, van Velthuysen ML, Rosenberg EH, Beijnen JH, Schellens JH, Cats A (2015) HPV-negative squamous cell carcinoma of the anal canal is unresponsive to standard treatment and frequently carries disruptive mutations in TP53. Br J Cancer 112: 1358-1366.

Rieckmann T, Tribius S, Grob TJ, Meyer F, Busch CJ, Petersen C, Dikomey E, Kriegs M (2013) HNSCC cell lines positive for HPV and p16 possess higher cellular radiosensitivity due to an impaired DSB repair capacity. Radiotherapy and Oncology 107(2): 242-246.

Rodel F, Wieland U, Fraunholz I, Kitz J, Rave-Frank M, Wolff HA, Weiss C, Wirtz R, Balermpas P, Fokas E, Rodel C (2014) Human papillomavirus DNA load and p16INK4a expression predict for local control in patients with anal squamous cell carcinoma treated with chemoradiotherapy. Int J Cancer 136: 278-288.

Rubio CA, Nilsson PJ, Petersson F, Hoog A, Blegen H, Chetty R (2008) The clinical significance of massive intratumoral lymphocytosis in squamous cell carcinoma of the anus. Int J Clin Exp Pathol 1: 376-380.

Serup-Hansen E, Linnemann D, Skovrider-Ruminski W, Hogdall E, Geertsen PF, Havsteen H (2014) Human papillomavirus genotyping and p16 expression as prognostic factors for patients with American Joint Committee on Cancer stages I to III carcinoma of the anal canal. J Clin Oncol 32: 1812-1817.

Stevanović S, Draper LM, Langhan MM, Campbell TE, Kwong ML, Wunderlich JR, Dudley ME, Yang JC, Sherry RM, Kammula US, Restifo NP, Rosenberg SA, Hinrichs CS (2015) Complete regression of metastatic cervical cancer after treatment with human papillomavirustargeted tumor-infiltrating T cells. J Clin Oncol 33: 1543-1550.

Ward MJ, Thirdborough SM, Mellows T, Riley C, Harris S, Suchak K, Webb A, Hampton C, Patel NN, Randall CJ, Cox HJ, Jogai S, Primrose J, Piper K, Ottensmeier CH, King EV, Thomas GJ (2014) Tumour-infiltrating lymphocytes predict for outcome in HPV-positive oropharyngeal cancer. Br J Cancer 110: 489-500.

Westra WH, Taube JM, Poeta ML, Begum S, Sidransky D, Koch WM (2008) Inverse relationship between human papillomavirus-16 infection and disruptive p53 gene mutations in squamous cell carcinoma of the head and neck. Clin Cancer Res 14: 366-369. 